\title{
STUDY OF CLINICAL PROFILE OF CHRONIC OBSTRUCTIVE PULMONARY DISEASE AND EVALUATION OF PULMONARY HYPERTENSION WITH ECG AND 2D ECHO FINDINGS
}

\author{
Basavaraj G. Mangshetty ${ }^{1}$, Kunireddy Apuroopa ${ }^{2}$ \\ 1 Professor, Department of General Medicine, M. R. Medical College, Kalaburagi. \\ 2Postgraduate Student, Department of General Medicine, M. R. Medical College, Kalaburagi.
}

\begin{abstract}
BACKGROUND

The recent definition by American Thoracic Society defines chronic obstructive pulmonary disease as a disease characterised by the presence of air flow obstruction due to chronic bronchitis or emphysema, which is generally progressive, maybe accompanied by airway hyperactivity and maybe partially reversible. In India, COPD is the second most common lung disorder after pulmonary TB. Tobacco smoking is by far the most important risk factor for COPD. Smoking cessation is the only intervention which has shown to slow down the progress of the disease. COPD should be considered in any patient presenting with cough, sputum production, or dyspnoea, especially if an exposure to risk factors for the disease has been present. Clinical diagnosis needs to be confirmed by standardised spirometric tests in the presence of incompletely reversible airflow limitation. Pulmonary arterial hypertension is the major cardiovascular complication of COPD and is associated with the development of Right ventricular hypertrophy (cor pulmonale) and is associated with poor prognosis. ECG is a simple bedside investigation, and right ventricular hypertrophy on ECG has a reasonably high specificity. Detectable tricuspid regurgitation by Echo Doppler is probably the best technique to measure pulmonary artery pressure noninvasively. Therefore, the present study is conducted to correlate the diagnostic values of ECG and Echo findings in COPD patients, which help in early detection of cardiac complications of chronic obstructive pulmonary disease giving time for early intervention.
\end{abstract}

\section{MATERIALS AND METHODS}

This was a single centre, hospital based, cross sectional study. COPD patients with cough, sputum production, dyspnoea were chosen. Pulmonary Function Test was done to pick up patients with reduced FEV $<80 \%$. They were nebulised with salbutamol bronchodilator and PFT was repeated to pick up patients with $<15 \%$ reversibility. All the data was tabulated and subjected to statistical analysis. The study was conducted in 72 patients admitted in Basaveshwar Teaching and General Hospital, Gulbarga attached to Mahadevappa Rampure Medical College from November 2014 to May 2016. Detailed history, thorough examination and routine investigations were done. The sputum was sent for routine examination, sputum for AFB negative confirmed and other investigations like electrocardiogram, echocardiogram, pulmonary function test were performed.

\section{RESULTS}

Among 72 patients, 43 patients were smokers and 24 patients' ECGs showed P- pulmonale. 41 patients had right axis deviation, 7 had R- wave height in lead V1 more than $5 \mathrm{~mm}$ (indicating they had severe right ventricular hypertrophy or pulmonary hypertension). Out of 72 patients, 12 had P wave amplitude in lead II+III+aVF > $9 \mathrm{~mm}$. Out of 72 patients, 12 had coronary artery disease. Out of 72 patients, 15 had pulmonary hypertension echocardiographically. 13 out of 72 had tricuspid regurgitation which is a non-invasive method of pulmonary artery pressure measurement.

\section{CONCLUSION}

Pulmonary hypertension was the most common cardiovascular complication leading to cor pulmonale, and emphysema was more common in smokers, bronchitis was more common in females, the prevalence of COPD was more in urban areas due to pollution of air by automobiles.

\section{KEYWORDS}

COPD, Pulmonary Hypertension; Cor Pulmonale, Emphysema.

HOW TO CITE THIS ARTICLE: Mangshetty BG, Apuroopa K. Study of clinical profile of chronic obstructive pulmonary disease and evaluation of pulmonary hypertension with ECG and 2D Echo findings. J. Evolution Med. Dent. Sci. 2016;5(100):7331-7336, DOI: $10.14260 /$ jemds/2016/1660

Financial or Other, Competing Interest: None.

Submission 10-11-2016, Peer Review 04-12-2016,

Acceptance 10-12-2016, Published 15-12-2016.

Corresponding Author:

Dr. Kunireddy Apuroopa,

Room No. 5 ,

P. G. Women's Residential Hostel,

Basaveshwar Hospital, Sedam Road,

Kalaburagi-585105.

E-mail:appulibra2@gmail.com

DOI: $10.14260 /$ jemds/2016/1660

\section{(c) $(i)(2)$}

\section{BACKGROUND}

Chronic obstructive pulmonary disease (COPD) is defined as a disease state characterised by airflow limitation that is not fully reversible. ${ }^{1}$

The three most common symptoms in COPD are cough, sputum production, and exertional dyspnoea.

COPD includes emphysema, an anatomically defined condition characterised by destruction and enlargement of the lung alveoli; chronic bronchitis, a clinically defined condition with chronic cough and phlegm; and small airways disease, a condition in which small bronchioles are narrowed. 
Typically, they have reduced FEV1 and reversibility less than 15 percent while bronchial asthma patients have more than 15 percent reversibility.

COPD prevalence in the world is influenced predominantly by smoking and shows age-specific GOLD categories of COPD stratified by smoking status, and shows that a high proportion of people aged 65 years have evidence of COPD.

Hypersecretion of mucus is a symptom that has been extensively studied in general population surveys over the last 40 years. In these studies, usually in middle-aged men, the prevalence of chronic cough and the production of sputum ranges between 15 and 53\%, with a lower prevalence of 8$22 \%$ in women, being more prevalent in urban than rural areas. Smoking low-tar cigarettes results in less cough and phlegm than smoking high-tar cigarettes. ${ }^{2}$

Impaired lung function is an excellent predictor of both morbidity and mortality, including the development of lung cancer, elevated C-reactive protein levels, osteoporosis and death. In an analysis of participants in the first National Health And Nutritional Examination Survey (NHANES) and followup, current and former smokers with GOLD stage-3 or 4 COPD had a significantly increased mortality risk whereas never-smoker with GOLD stage-3 or 4 COPD did not have an increased mortality risk. Celli et al demonstrated an index that includes body mass, dyspnoea and the $6 \mathrm{~min}$. walk along with lung function, which is a much better predictor for mortality than lung function alone.

Prevalence studies of COPD are normally based on values of percentage predicted forced expiratory volume in $1 \mathrm{~s}$ $\left(\mathrm{FEV}_{1}\right)$, which defines individuals with and without airways obstruction. In current smokers in the age range 40-65, 18\% of men had an $\mathrm{FEV}_{1}$ greater than 2 SD below normal and $14 \%$ of women compared with $7 \%$ and $6 \%$ of non-smokers respectively.

National surveys of consultations in British general practices have shown the changes in prevalence of clinically diagnosed chronic bronchitis, emphysema and COPD over the last 40 years. These data show a modest decline in the number of middle-aged men with symptoms suggestive of COPD and a slight increase among middle-aged women.

According to World Health Organization estimates, 65 million people have moderate to severe COPD. More than 3 million people died of COPD in 2005 corresponding to $5 \%$ of all deaths globally and it is estimated to be the third leading cause of death by 2030 .

The exact incidence of clinically significant pulmonary hypertension, defined as pulmonary hypertension that contributes to symptomatology and prognosis, is difficult to estimate in COPD. A prevalence of $10-30 \%$ in patients with at least one previous hospitalisation seems reasonable. ${ }^{3}$

The mortality of patients while hospitalised for a COPD exacerbation is approximately $5 \%$ to $14 \% .^{4}$

In India, COPD is the second most common lung disorder after pulmonary TB. And it is one of the leading causes of death in the high income countries also.

The prevalence and mortality data greatly underestimates the total burden of COPD as the disease is usually not diagnosed until it is clinically apparent and moderately advanced. Tobacco smoking is by far the most important risk factor for COPD. Smoking cessation is the only intervention which has shown to slow down the progress of the disease.

COPD should be considered in any patient presenting with cough, sputum production, or dyspnoea, especially if an exposure to risk factors for the disease has been present. Clinical diagnosis needs to be confirmed by standardised spirometric tests in the presence of incompletely reversible airflow limitation.

The major morbidity of COPD is due to the impact on cardiac performances which is directly due to pulmonary arterial hypertension.

Pulmonary arterial hypertension develops late in the course of COPD with the development of hyperaemia (PaO2 $<60 \mathrm{mmHg}$ ) and hypercapnia. It is the major cardiovascular complication of COPD and is associated with the development of Right ventricular hypertrophy (cor pulmonale) and is associated with poor prognosis.

ECG is a simple bedside investigation. ECG criteria for detecting right ventricular hypertrophy have a reasonably high specificity. Detectable tricuspid regurgitation by Echo Doppler is probably the best technique to measure pulmonary artery pressure noninvasively. Therefore, the present study is conducted to correlate the diagnostic values of ECG and Echo findings in COPD patients, which help in early detection of cardiac complications of chronic obstructive pulmonary disease giving time for early intervention.

\section{MATERIALS AND METHODS}

The study was conducted in 72 patients admitted in Basaveshwar Teaching and General Hospital, Gulbarga attached to Mahadevappa Rampure Medical College from November 2014 to May 2016. Detailed history, thorough examination and routine investigations were done. The sputum was sent for routine examination, sputum for AFB negative confirmed and other investigations like electrocardiogram, echocardiogram, pulmonary function tests were performed.

\section{Selection Criteria}

Step 1- The patients with cough, sputum production, dyspnoea are chosen and sputum AFB negative is confirmed.

Step 2- Pulmonary function test was done to pick up patients with reduced FEV $<80 \%$.

Step 3- Nebuliser with salbutamol bronchodilator and PFT was done to select patients with $<15 \%$ reversibility.

Step 4- They were clinically examined for over inflation, ascites, oedema, parasternal heave, JVP, loud P2 and tricuspid regurgitation murmur.

Step 5- X-ray chest PA view, lateral view taken to rule out the right pulmonary artery dilatation $>16 \mathrm{~mm}$ [characteristic of pulmonary arterial hypertension], lateral view to rule out retrosternal space obliteration $[\mathrm{RVH}]$.

Step 6- ECG was taken in patients to look for $\mathrm{p}$ wave morphology, amplitude in lead II, III, aVF. P wave amplitude $>9$ $\mathrm{mm}, \mathrm{P}$-axis, R-wave $>5 \mathrm{~mm}, \mathrm{r} / \mathrm{s}>50 \%$ and RBBB.

Step 7- Echocardiogram was done for the chosen patients. Pulmonary arterial hypertension and tricuspid regurgitation was looked for [as it is the noninvasive measure of pulmonary arterial pressure, a surrogate for measuring PAP]. 
Patients were interviewed for the symptoms like cough, dyspnoea, wheeze. They were examined to look for signs of pulmonary hypertension and cor pulmonale.

\section{Exclusion Criteria}

- Bronchial asthma.

- Pulmonary Tuberculosis.

- Interstitial lung disease.

- Valvular heart disease.

- Acute Left Ventricular Failure and pulmonary oedema secondary to other causes (hypertension, ischaemic heart disease, cardiomyopathies).

- Primary pulmonary hypertension.

- Bronchiectasis.

Data was collected using a pretested proforma meeting the objectives of the study. Detailed history, physical examination and necessary investigations were done. They were examined for over inflation, ascites, oedema, parasternal heave, raised JVP, loud P2, tricuspid regurgitation murmur.

Using noninvasive methods like ECG, Chest X-ray, 2D Echocardiography, pulmonary hypertension was evaluated in COPD patients.

ECG was taken to look for P wave morphology and P was amplitude in lead II, III and aVF and also for $\mathrm{P}$ wave axis, $\mathrm{R}$ wave amplitude in chest leads from V1 to V6 and RBBB.

Echocardiogram was done to look for pulmonary artery hypertension and tricuspid regurgitation as it is a noninvasive measure of pulmonary arterial pressure. The analysis of the data was done using appropriate statistical methods.

\section{Study Design}

Cross sectional study.

\section{Ethics}

The procedures followed were in accordance with the ethical standards of the responsible committee on human experimentation.

The purpose of the study was explained to the patient and informed consent obtained.

\section{Statistical Analysis}

This was a single centre, hospital based, cross sectional study.

All the data was tabulated and subjected to statistical analysis. Descriptive statistics were used, such as mean, SD and proportion and other appropriate statistical tests were used.

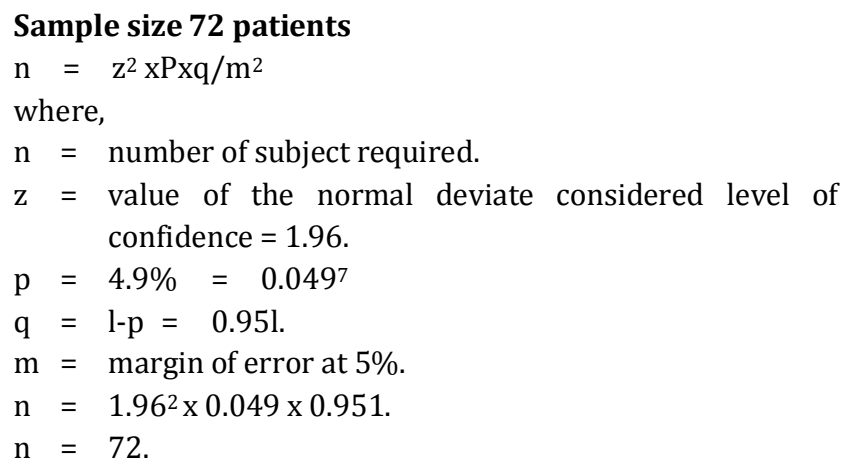

Sample size 72 patients

\section{RESULTS}

72 COPD patients were studied from November 2014 to May 2016.

Among 72 patients, 43 patients were smokers and 24 patients' ECGs showed P-pulmonale. 41 patients had right axis deviation, 7 had $\mathrm{R}$ wave height in lead V1 more than $5 \mathrm{~mm}$ (Indicating they had severe right ventricular hypertrophy or pulmonary hypertension).

Out of 72 patients, 12 had $\mathrm{P}$ wave amplitude in lead II + III $+\mathrm{aVF}>9 \mathrm{~mm}$, this is an indication for life long oxygen therapy according to American Thoracic Society (ATS).

Out of 72 patients, 12 had coronary artery disease, this increases the incidence of cor pulmonale.

Out of 72 patients, 15 had pulmonary hypertension echocardiographically.

13 out of 72 had tricuspid regurgitation which is a noninvasive method of pulmonary artery pressure measurement.

Out of 72 patients, $21 \%$ developed pulmonary hypertension, 18\% developed tricuspid regurgitation, 34\% had $\mathrm{P}$ - pulmonale, $18 \%$ had $\mathrm{P}$ wave amplitude $>9 \mathrm{~mm}$ in lead II+III+aVF. $18 \%$ had concomitant coronary artery disease.

This is important as systemic inflammation increases chances of atherosclerosis.

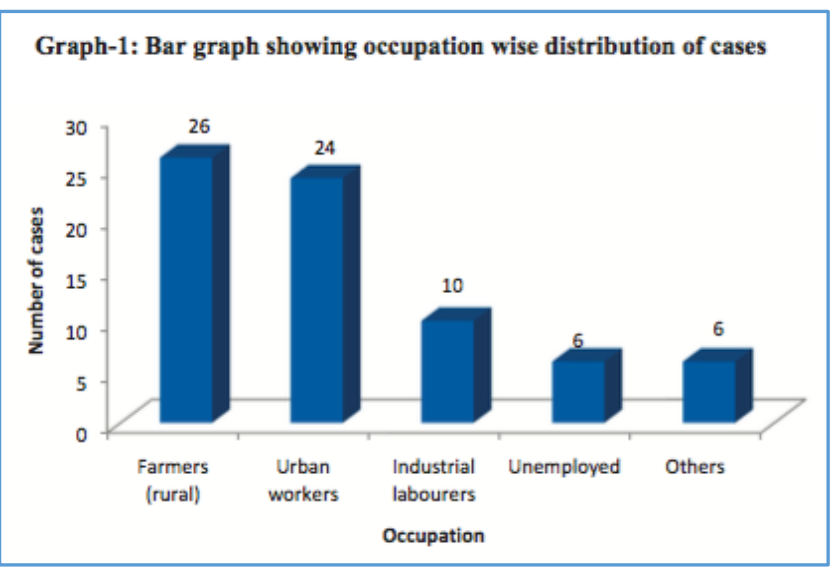

Graph-2: Pie chart showing sex wise distribution of cases

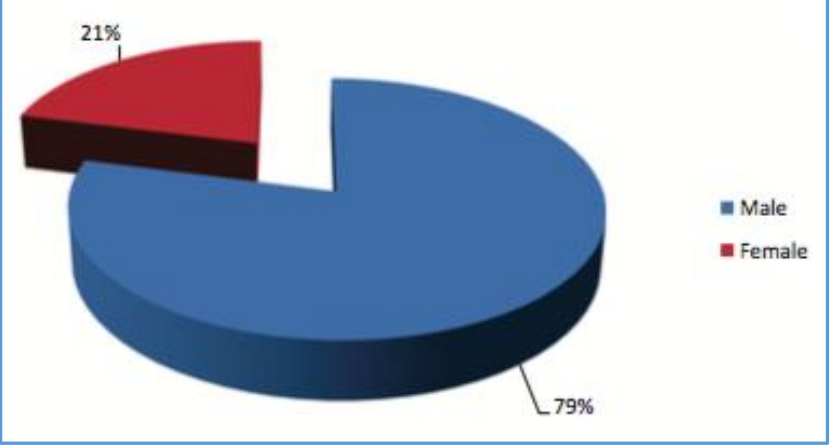



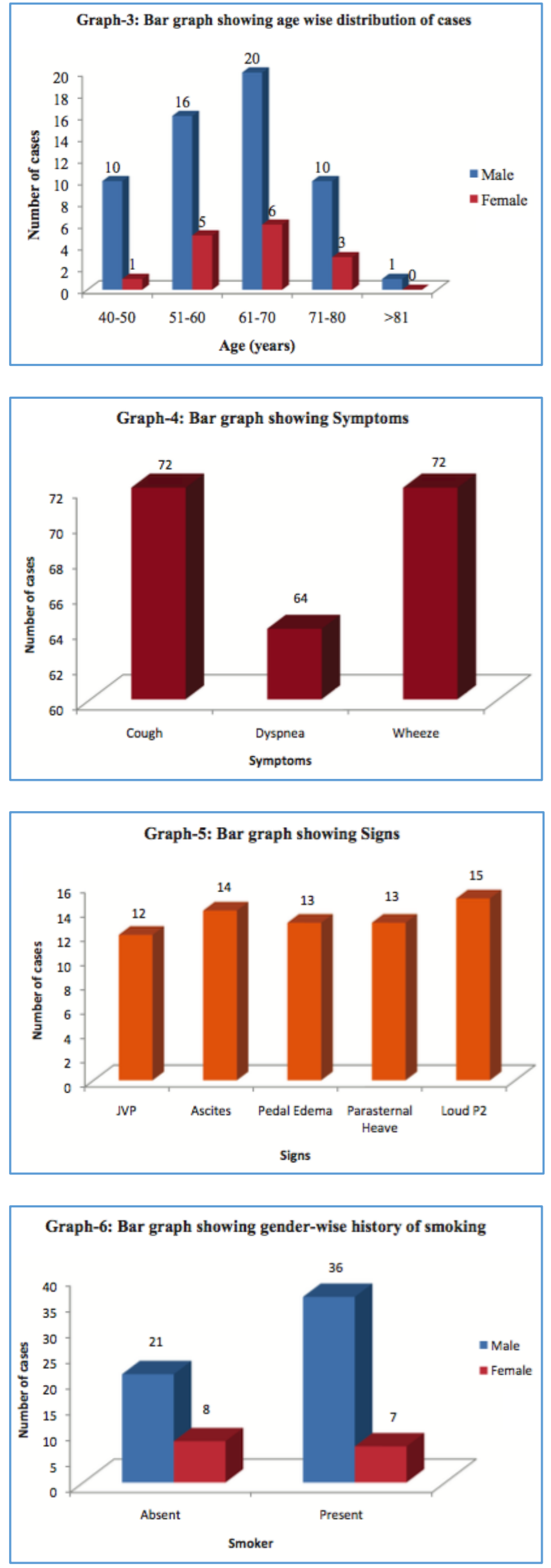
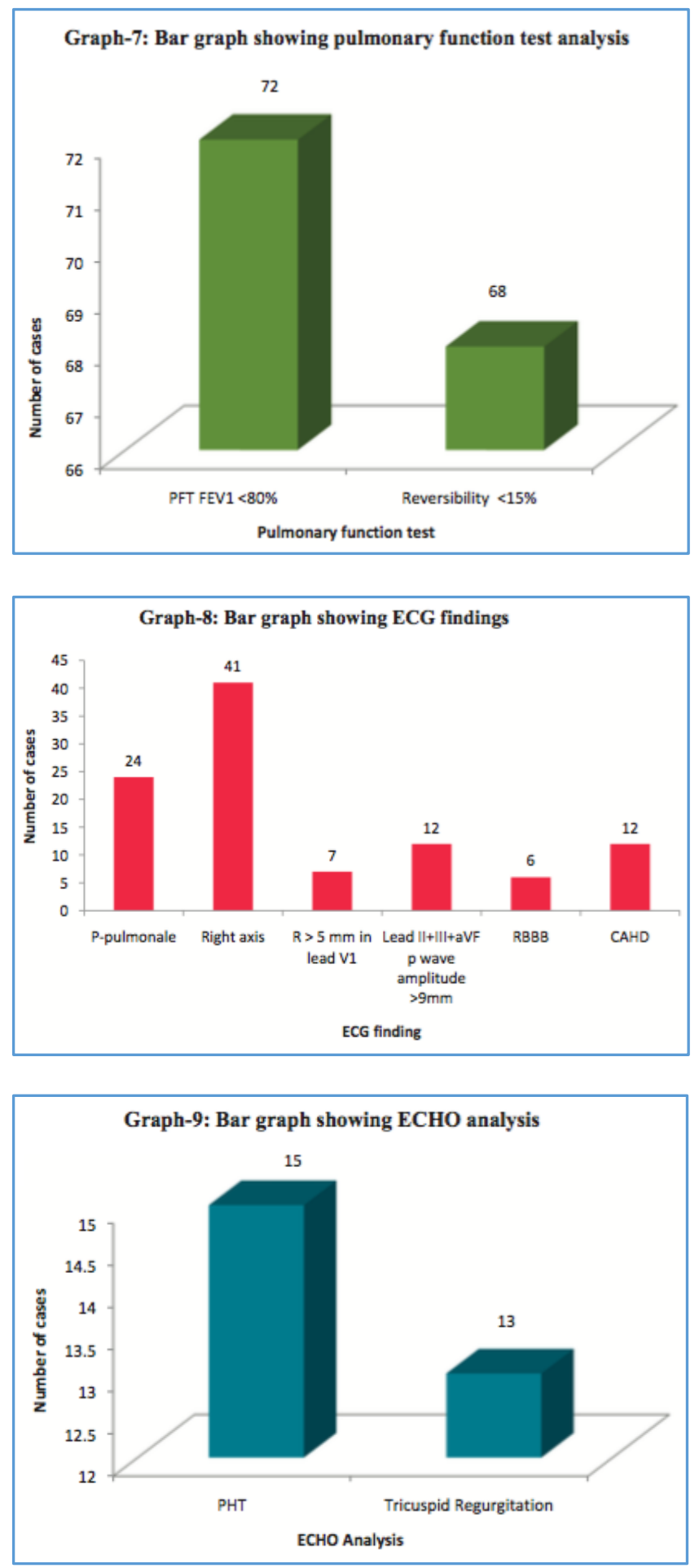

\begin{tabular}{|c|c|}
\hline Stage & Spirometric Findings \\
\hline \multirow{2}{*}{ I: Mild } & FEV1/FVC $<0.70$ \\
\cline { 2 - 2 } & FEV1 $>80 \%$ predicted \\
\hline \multirow{2}{*}{ II: Moderate } & FEV1 $/ \mathrm{FVC}<0.70$ \\
\cline { 2 - 2 } & $50 \%<\mathrm{FEV} 1<80 \%$ predicted \\
\hline \multirow{2}{*}{ III: Severe } & FEV1/FVC $<0.70$ \\
\cline { 2 - 2 } & $\begin{array}{c}\text { FEV1 }<30 \% \text { predicted or FEV1 }<50 \% \\
\text { predicted plus chronic respiratory } \\
\text { failure }\end{array}$ \\
\cline { 2 - 2 } & $\begin{array}{c}\text { Fevere } 1 / \mathrm{FVC}<0.70 \\
\text { Table 1. Assessment of Severity (GOLD) }\end{array}$ \\
\hline
\end{tabular}




\begin{tabular}{|c|c|}
\hline X-ray & Number of Cases \\
\hline $\begin{array}{c}\text { X-ray PA View right ventricular } \\
\text { hypertrophy (RVH) }\end{array}$ & $16 / 72$ \\
\hline $\begin{array}{c}\text { Lateral view right ventricular } \\
\text { hypertrophy (RVH) }\end{array}$ & $16 / 72$ \\
\hline \multicolumn{2}{|c|}{ Table 1. X-ray Analysis } \\
\hline
\end{tabular}

\section{DISCUSSION}

Worldwide, the most commonly encountered risk factor for COPD is tobacco smoking. Outdoor, occupational, and indoor air pollution - the latter resulting from the burning of biomass fuels - are other major COPD risk factors. Nonsmokers may also develop COPD. ${ }^{2}$

The genetic risk factor that is best documented is a severe hereditary deficiency of alpha-1 antitrypsin.

Male patients with smoking history were more prone to COPD, in particular emphysema.

In smokers working in textile industries, bronchitis was more common, while smoking commonly produced central acinar emphysema. In textile industry workers, the fine fabric related chemical particles increased the incidence of bronchitis in these patients.

Emphasis should be on primary prevention, which is best achieved by elimination or reduction of exposures to various substances in the workplace. Secondary prevention, achieved through surveillance and early detection, is also important. ${ }^{2}$

This indicates the increased level of pollution in urban areas. 36 men with COPD were smokers. Major air pollutants were released from automobiles like carbon monoxide, carbon dioxide, sulphur dioxide, lead, so urbanisation had its toll by increasing the level of pollutants in the air and increasing the number of COPD patients. Strict pollution control measures should be implemented immediately.

From the above data we can infer that the rapid pace of urbanisation, air pollution, farming activities and smoking are major triggers for COPD.

Moreover, the complications of COPD like pulmonary hypertension was detected by ECG and ECHO.

The chronic hypoxia of COPD was responsible for the development of pulmonary hypertension. The tricuspid regurgitation was the best possible noninvasive way of measuring pulmonary artery pressure.

$18 \%$ patients had concomitant coronary artery disease, this observation is important, because systemic inflammation enhances atherosclerosis and diabetes. TNF alpha is increased in COPD patients.

Pulmonary hypertension was the major cardiovascular complication of COPD, the chronic hypoxia of COPD is responsible for the development of pulmonary hypertension. COPD-related PH is usually mild. Small subset of patients demonstrates significant $\mathrm{PH}$ and the clinical significance is limited to poor prognosis. Initial goal of management is to identify alternate remediable causes. There is no current therapy targeting PH in COPD.

Smoking cessation and oxygen therapy remain the only proven therapies for COPD and should be recommended to all COPD patients. Anecdotal reports suggest utility of PDE5inhibitor and prostacyclin in treatment of $\mathrm{PH}$ associated with COPD. 5

Approximately, $10 \%-30 \%$ of patients with moderate to severe COPD have elevated pulmonary pressures. When COPD is associated with $\mathrm{PH}$, both mortality and morbidity are increased. There are no clinical or physical examination findings that accurately identify patients with underlying $\mathrm{PH}$. Radiographic imaging findings are specific but not sensitive indicators of $\mathrm{PH}$. Echocardiography is the principle noninvasive diagnostic test but may be technically limited in a significant proportion of patients with COPD. Right heart catheterisation is required for accurate measurement of pulmonary pressures. The combined effects of inflammation, endothelial cell dysfunction, and angiogenesis appear to contribute to the development of PH associated with COPD. Systemic vasodilators have not been found to be effective therapy. Selective pulmonary vasodilators, including inhaled nitric oxide and phosphodiesterase inhibitors, are promising treatments for patients with COPD associated PH. 6

The study also established that the best noninvasive way to detect raised pulmonary arterial pressure was detecting tricuspid regurgitation by echocardiography.

Males predominantly had emphysema, and females predominantly had chronic bronchitis with cor pulmonale.

Urbanisation, pollution of air, industrialisation, farming activities and smoking are major triggers for COPD. The complications of COPD like pulmonary hypertension and cor pulmonale were better detected by ECG and echocardiogram.

Finally, the study by analysing COPD patients with clinical parameters, ECG, echocardiogram, concluded that pulmonary hypertension was the most common cardiovascular complication leading to cor pulmonale. Emphysema was more common in smokers, bronchitis was more common amongst women and the prevalence of COPD was more in urban areas due to pollution of air by automobiles. The textile based industries were mainly responsible for the increased prevalence of COPD.

This is currently the fourth most common cause of death both in the US and worldwide. To date, the only proven costeffective therapies for the disease are the cessation or prevention of smoking, which is the single most common cause of COPD, and vaccination to prevent influenza and pneumococcal infection. Hospitalisation and associated costs represent the greatest healthcare expenditures for people with the disease. Long-term oxygen therapy is also among the costliest interventions for COPD treatment, but oxygen therapy is the only intervention to date that has been shown to decrease death rates due to COPD. ${ }^{7}$

Current drug therapy for stable COPD focuses primarily on bronchodilation through inhaled beta2-agonists and anticholinergic agents, immunisation, and elimination of smoking as a risk factor. Although many pharmacologic agents are available to treat COPD, no drug has demonstrated effectiveness in halting progression of the disease. ${ }^{8}$ Studies demonstrated no clinically significant advantage to antimicrobial therapy. ${ }^{9}$ Microorganisms causing acute exacerbations of COPD are not only typical bacteria (46\%) but also atypical pathogens (26\%). ${ }^{10}$ There was no difference in outcome between older traditional antibiotics with adequate coverage against organisms associated with acute exacerbation of chronic bronchitis and newer broad-spectrum antibiotics. ${ }^{11}$ But some studies suggest a small but statistically significant improvement due to antibiotic therapy in patients with exacerbations of COPD. ${ }^{12}$

Several factors such as environmental exposures and viral or bacterial infections have been linked to the acute worsening of COPD patients. What proportion of AECBs are caused by bacterial infection remains largely uncertain and therefore the place of antibiotics is still being debated. This represents a 
crucial medical and economic problem. ${ }^{13}$

The cellular and molecular mechanisms by which viruses cause exacerbations remain undetermined. Future research efforts to understand these mechanisms would aid the development of novel therapeutics to reduce the morbidity and mortality of this disease. 14

Patients with stable hypoxaemic COPD have an impaired ability to excrete sodium which is not improved by the administration of an ACE inhibitor. This suggests that the inability of patients with hypoxaemic COPD to excrete sodium is not caused by their increased plasma levels of aldosterone. 15

Smoking education is the most important fact in the care of COPD. The patient needs to be demonstrated on the proper use of inhalers, home management of medications, avoidance of respiratory irritations. Pulmonary rehabilitation programs, exercise programs tailored to the severity of disease, can improve the patient's functional status, exercise tolerance, and the symptoms of breathlessness. ${ }^{16}$

\section{ACKNOWLEDGEMENT}

It gives us immense pleasure to express our gratitude and sincere thanks to Dr. Suresh M Chincholi, Professor and HOD of Department of Medicine, M. R. Medical College, who has been a constant source of inspiration and encouragement. Our sincere thanks to Dr. Kumarswamy, Dr. Suresh Harsoor, Dr. Basavaraj Belli, Dr. Sangram Biradar, Dr. B Raikod Patil, Dr. Satish Kinagi, Dr. Shoukat A R, Dr. Murugesh Pastapur, Dr. Bharat Konin, and to all staff members of Department of Medicine.

\section{CONCLUSION}

From this study, we have found out that smoking was the main risk factor of COPD. Occupational exposure played an important role in nonsmokers. Pulmonary Hypertension was found in $20 \%$ of the individuals which can be detected early with ECG and 2D Echo. Immediate measures such as smoking cessation, bronchodilators and oxygen therapy proved to be beneficial.

\section{REFERENCES}

1. Reilly JJ, Silverman EK, Shapiro SD. Harrisons textbook of internal medicine. Chapter 314. 19th edn. McGraw Hill Professional. USA 2015;2:1700.

2. MacNee W. Chronic bronchitis and emphysema. Chapter 23. In: Seaton A, Seaton D. Crofton and Douglas respiratory diseases. $5^{\text {th }}$ edn. Vol.1. Blackwell Science Ltd 2000:p 616.
3. Naeije R. Pulmonary hypertension and right heart failure in chronic obstructive pulmonary disease. Proceedings of the American Thoracic Society 2005;2(1):20-2.

4. Siedman JC. Chronic obstructive pulmonary disease. In: Tintinalli J (edr). Emergency medicine: a comprehensive study guide. McGraw-Hill Corn 1992:298-302.

5. Jyothula S, Safdar Z. Update on pulmonary hypertension complicating chronic obstructive pulmonary disease. Int J Chron Obstruct Pulmon Dis 2009;4:351-63.

6. Elwing J, Panos RJ. Pulmonary hypertension associated with COPD. Int J Chron Obstruct Pulmon Dis 2008;3(1):55-70.

7. Faulkner MA, Hilleman DE. The economic impact of chronic obstructive pulmonary disease. Expert Opin Pharmacother 2002;3(3):219-28.

8. Faulkner MA, Hilleman DE. Pharmacologic treatment of chronic obstructive pulmonary disease: past, present, and future. Pharmacotherapy 2003;23(10):1300-15.

9. Hirschmann JV. Do bacteria cause exacerbations of COPD? Chest 2000;118(1):193-203.

10. Lieberman D, Lieberman D, Ben-Yaakov $M$, et al. Infectious etiologies in acute exacerbation of COPD. Diagn Microbiol Infect Dis 2001;40(3):95-102.

11. Peng CC, Aspinall SL, Good CB, et al. Equal effectiveness of older traditional antibiotics and newer broad-spectrum antibiotics in treating patients with acute exacerbations of chronic bronchitis. South Med J 2003;96(10):986-91.

12. Saint $S$, Bent $S$, Vittinghoff $E$, et al. Antibiotics in chronic obstructive pulmonary disease exacerbations. A metaanalysis. JAMA 1995;273(12):957-60.

13. Sohy C, Pilette C. Acute exacerbation of chronic obstructive pulmonary disease and antibiotics: what studies are still needed? European Respiratory Journal 2002;19:966-75.

14. Varkey AB. Chronic obstructive pulmonary disease in women: exploring gender differences. Curr Opin Pulm Med 2004;10(2):98-103.

15. Stewart AG, Waterhouse JC, Billings CG, et al. Effects of angiotensin converting enzyme inhibition on sodium excretion in patients with hypoxemic chronic obstructive pulmonary disease. Thorax 1994;49(10):995-8.

16. Rosen P. Dailey RH: Chronic obstructive pulmonary disease. In: emergency medicine concepts and clinical practice. $3^{\text {rd }}$ edn. Mosby-Year Book mc 1992:1093-1111. 